\title{
A Note on Detecting Algebraic Cycles
}

\author{
G. V. Ravindra
}

Abstract. The purpose of this note is to show that the homologically trivial cycles contructed by Clemens and their generalisations due to Paranjape can be detected by the technique of spreading out. More precisely, we associate to these cycles (and the ambient varieties in which they live) certain families which arise naturally and which are defined over $\mathbb{C}$ and show that these cycles, along with their relations, can be detected in the singular cohomology of the total space of these families.

\section{Introduction}

One of the fundamental questions in the theory of algebraic cycles is their detection. More precisely, given an algebraic cycle, one would like to know when its class in the Chow group of cycles modulo rational equivalence is non-trivial. The usual way of detecting cycles is purely cohomological i.e., via their images under the cycle class map and the Abel-Jacobi map.

The conjectural framework of mixed motives provides an answer to this question via the existence of filtrations on the Chow groups and higher Abel-Jacobi maps [6]. Filtrations satisfying many of these properties has been constructed by Asakura [1], Lewis [8], Paranjape [2], and Saito [12], among others.

The fundamental idea underlying the above constructions is as follows. A variety $V$ over $\mathbb{C}$ can be thought of as a family of varieties over the algebraic closure $\overline{\mathbb{O}} \mathcal{L} \subset$ $\mathbb{C}$ of the field of rational numbers. Even when the variety is defined over $\mathbb{O}$, the Chow group of such a variety (when considered over $\mathbb{C}$ ) may contain cycles that are defined over larger fields. In particular, the usual examples of non-trivial elements in $F^{2} \mathrm{CH}^{2}(S)$ where $S$ is a surface are defined over fields of transcendence degree 2 [13]. Thus, in order to detect such cycles we must use the full force of such a "family"-like structure.

For any variety $V$ over $\mathbb{C}$ we consider the collection of Cartesian diagrams

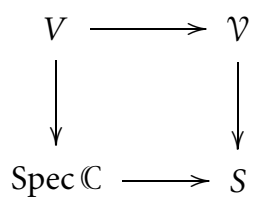

where $S$ and $\mathcal{V}$ are varieties defined over $\overline{\mathbb{O}}$, and the lower horizontal arrow factors through the generic point of $S$. Assume for the moment that $V$ is smooth projective,

Received by the editors August 9, 2004; revised November 17, 2004.

AMS subject classification: $14 \mathrm{C} 25$.

(C) Canadian Mathematical Society 2006. 
and that $S$ and $\mathcal{V}$ are smooth and $\mathcal{V} \rightarrow S$ is proper and smooth. Since any algebraic cycle on $V$ (and $V$ itself) is defined over some finitely generated field, we have [7],

$$
\mathrm{CH}^{p}(V)=\underset{\lim }{\longrightarrow} H^{p}(\mathcal{V})
$$

There is a cycle class map into Deligne-Beilinson cohomology, see [5],

$$
\mathrm{CH}^{p}(\mathcal{V}) \rightarrow \mathrm{H}_{D b}^{2 p}(\mathcal{V},(\mathbb{O}(p))
$$

which when restricted to cycles homologous to zero can be identified with the usual Abel-Jacobi map. On taking limits, we get a map

$$
\mathrm{cl}_{\mathrm{ADb}}^{p}: \mathrm{CH}^{p}(V) \rightarrow \mathrm{H}_{\mathrm{ADb}}^{2 p}(V, \mathbb{O}(p))
$$

where the group on the right is defined to be the limit of the Deligne-Beilinson cohomology groups of the various models of $V$. One expects (this again follows from the Bloch-Beilinson conjectures) that cycles defined over fields of positive transcendence degree are detected by the above map. Since the above map is obtained by taking direct limits, we should be able to detect any given cycle at some finite stage, i.e., on some model $\mathcal{V}$.

The purpose of this note is to show that the cycles contructed by Clemens [4] and their generalisations due to Paranjape [10] can be detected by the technique of spreading out.

In the next section we analyse certain cycles which can be thought of as natural generalisation of the Clemens-Paranjape cycles. We associate to these cycles (and the ambient varieties in which they live) certain families (which are defined over $\mathbb{C}$ ) which arise naturally, and show that these cycles can be detected in the singular cohomology of the total space of these families. The advantage of working with families such as these is that we can now work with cohomology with $\mathbb{Z}$ coefficients. This makes it possible then to detect cycles whose image under the cycle class map is torsion. This can then be used to detect cycles along with their relations.

\section{The Cycles of Clemens and Paranjape}

\subsection{The Setup and Notation}

Let $X$ be a smooth projective variety of dimension $2 d-1$ and let $C$ be a codimension $d$ subvariety of $X$. Let $f: X \rightarrow S$ be a flat family where $X, S$ are smooth with $X$ as the geometric generic fibre. Further suppose that $S$ is a curve and the family has special fibre $X_{0}$ over $s_{0} \in S$ containing only ordinary double points $\left\{p_{i}\right\}$ as singularities. Let $\mathcal{C} \rightarrow T$ be a smooth family with geometric generic fibre $C$ such that there exists a diagram:

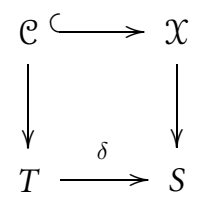


Assume that the map $T \rightarrow S$ is a finite cover with simple ramification at $t_{0} \in T$ where $\delta\left(t_{0}\right)=s_{0}$.

We consider the following two cases.

(i) The special fibre $C_{0}$ of the family $\mathcal{C}$ passes through exactly one of the double points, denoted $p_{0}$, in the fibre $X_{0}$.

(ii) The special fibre $C_{0}$ of the family $\mathcal{C}$ misses the double points in the fibre $X_{0}$.

Let $\tilde{X}_{0}$ be the blow up of the double point $p_{0}$ and let $E$ denote the exceptional fibre over the point $p_{0}$.

Let $X_{T}:=X \times_{S} T$ be the pull back of $\mathcal{X}$ to a family over $T$. $X_{T}$ then picks up ordinary double point singularities at the singular points of $X_{0}$. By the universal property of fibre products there is a lifting $\tilde{i}: \mathcal{C} \hookrightarrow X_{T}$.

Let $y \rightarrow X_{T}$ be the blowing up at the ordinary double point $p_{0}$. The special fibre of $y \rightarrow T$ at $t_{0}$ is the union of $\tilde{X}_{0}$ and a smooth quadric $Q$ such that $\tilde{X}_{0}$ meets $Q$ transversally along $E$. Let $\tilde{\mathcal{C}}$ be the strict transform under the blow up map. If $C_{0}$ passes through the double point $p_{0}$ then $\tilde{\mathcal{C}}$ intersects $Q$ in a projective space $\mathbb{P}^{d-1} \subset E$.

We make the following assumption in order to simplify our arguments.

Assumption 2.1 For the generic fibre $X$ in the family $X \rightarrow S, \mathrm{H}^{2 d}(X, \mathbb{Z}) \cong \mathbb{Z}$ so that the composite map (for the given embedding $X \hookrightarrow \mathbb{P}^{N}$ )

$$
\mathrm{CH}^{d}(X) \rightarrow \mathrm{H}^{2 d}(X, \mathbb{Z}) \cong \mathrm{H}_{2 d-2}(X, \mathbb{Z}) \cong \mathrm{H}_{2 d-2}\left(\mathbb{P}^{N}, \mathbb{Z}\right) \cong \mathbb{Z}
$$

is the degree map.

Let $A$ be a relatively ample class on $X \rightarrow S$. Let $\mathcal{H}$ be $A^{d}$ and denote by $H$ its restriction to a general fibre of the family $X \rightarrow S$. Assume that the degree of $H$ denoted by $\operatorname{deg}(H)$ is odd. Then $\Xi=\operatorname{deg}(H)$. $\mathrm{C}-\operatorname{deg}(C) . \mathcal{H}$ is a cycle on $X_{T}$ whose restriction to the generic fibre $\xi:=\operatorname{deg}(H) . C-\operatorname{deg}(C) . H$ is a nullhomologous cycle. Let $\tilde{\mathcal{C}}$ be the strict transform of $\mathcal{C}$ under the map $y \rightarrow X_{T}$ and let $\tilde{\Xi}:=\operatorname{deg}(H) . \tilde{\mathcal{C}}-$ $\operatorname{deg}(C) \cdot \mathcal{H}$.

\subsection{Detecting Nullhomologous Cycles}

We shall now show that the cycle $\tilde{\Xi}$ can be detected in the cohomology of the total space $y$ in the case when $C_{0}$ passes through exactly one ordinary double point. Our method here is entirely topological and avoids any use of Hodge theory. We shall work locally over a disc $\Delta$ around the point $0 \in S$ in the base locus. We denote by $\tilde{\Delta}$ the component of the inverse image of $\Delta$ in $T$ containing $t_{0}$. Having reduced the situation to such a neighbourhood, we note that the special fibre $X_{0}$ is a deformation retract of the family $X \times_{s} \Delta \rightarrow \Delta$. Without loss of generality, we can assume that $X_{0}$ contains exactly one ordinary double point. We then have that in the blow up family $y \times_{T} \tilde{\Delta} \rightarrow \tilde{\Delta}, \tilde{X}_{0} \cup Q$ is a deformation retract of $y \times_{T} \tilde{\Delta}$.

Theorem 2.2 We work with notation as above. Let $\mathcal{N}:=(y \backslash Q) \times_{T} \tilde{\Delta}$. 
(i) Suppose that the special fibre $C_{0}$ passes through the ordinary double point $p_{0}$, then the image of $\tilde{\Xi}:=\operatorname{deg}(H) . \tilde{\mathrm{e}}-(\operatorname{deg} C) . \mathcal{H}$ is non-trivial 2-torsion in the cohomology of $\mathcal{N}$.

(ii) If $C_{0}$ does not pass through $p_{0}$ in $X_{0}$ then the class of $\tilde{\Xi}$ is trivial in the cohomology of $\mathcal{N}$.

Proof (i) Consider the following diagram:

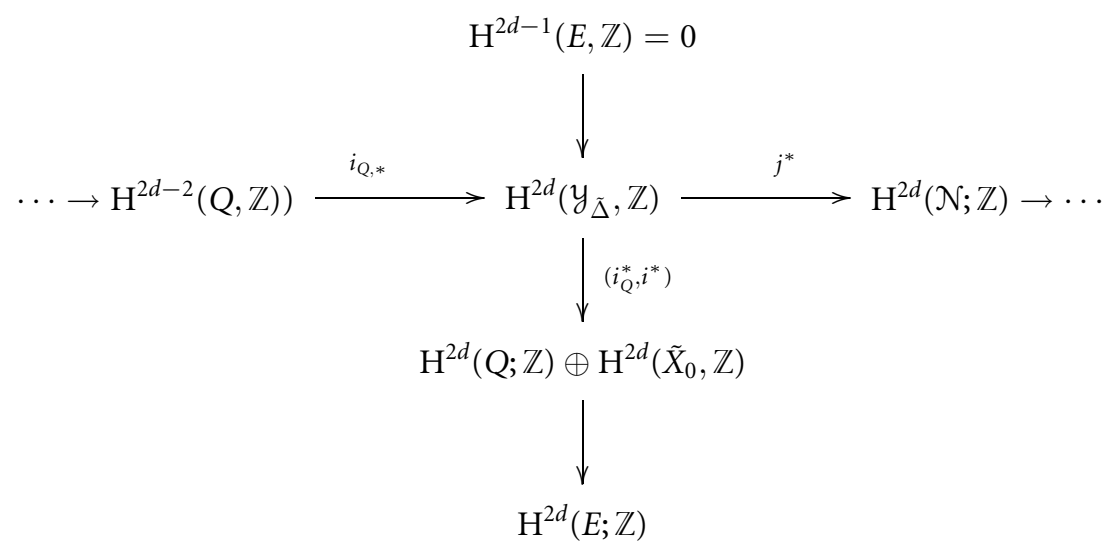

where the horizontal sequence is the Gysin sequence and the vertical one is the MayerVietoris sequence. The composite $i_{Q}^{*} \circ i_{Q, *}$ in the diagram

$$
\mathrm{H}^{2 d-2}(\mathrm{Q}, \mathbb{Z}) \stackrel{i_{Q, *}}{\longrightarrow} \mathrm{H}^{2 d}\left(\mathrm{y}_{\tilde{\Delta}}, \mathbb{Z}\right) \stackrel{i_{Q}^{*}}{\longrightarrow} \mathrm{H}^{2 d}(\mathrm{Q}, \mathbb{Z})
$$

is $\cup c_{1}\left(N_{Q / y}\right)$, the cup product with the first Chern class of the normal bundle of $Q$ in $y$. Now, $N_{Q / y} \cong \mathcal{O}_{Q}(-1)$ for the natural embedding $Q \hookrightarrow \mathbb{P}^{22}$. Since $c_{1}\left(\mathcal{O}_{Q}(1)\right)$ generates $\mathrm{H}^{2}(Q, \mathbb{Z})$, the first Chern class of this normal bundle can be identified with the class $-[E]$ where $[E]$ is the class of the exceptional fibre in $\tilde{X}_{0}$.

Furthermore, as $Q$ occurs naturally as a smooth odd-dimensional quadric hypersurface of $\mathbb{P}^{2 d}$, all its even cohomology groups are isomorphic to $\mathbb{Z}$.

Let $L \subset \tilde{\mathcal{C}}$ be the exceptional fibre. This is contained in $Q$ as a $\mathbb{P}^{p d-1}$ and therefore $[L]$ generates $\mathrm{H}^{2 d}(Q, \mathbb{Z})$. Thus the class of $\tilde{\Xi}$, denoted by $[\tilde{\Xi}]$, restricts to the class $\operatorname{deg}(H) \cdot[L]$ in $\mathrm{H}^{2 d}(Q, \mathbb{Z})$. Moreover its image under the isomorphism $\mathrm{H}^{2 d}\left(y_{\tilde{\Delta}}\right) \cong$ $\mathrm{H}^{2 d}\left(\tilde{X}_{0} \cup Q\right)$ is the class $\operatorname{deg}(H) .[L]$. On the other hand, since the composition $i_{Q}^{*} \circ$ $i_{Q, *}=\bigcup c_{1}\left(\mathcal{O}_{Q}(-1)\right.$ one has by the projection formula,

$$
\eta \cup i_{Q}^{*} c_{1}\left(\mathcal{O}_{\mathbb{P}^{2 d}}(-1)\right)=i_{Q}^{*}\left(i_{Q, *}(\eta) \cup c_{1}\left(\mathcal{O}_{\mathbb{P}^{2 d}}(-1)\right)\right)
$$

Now $Q$ does not contain any $d$-dimensional (or codimension $d-1$ ) linear subspace. Therefore when $\eta$ is the generator of $\mathrm{H}^{2 d-2}(Q, \mathbb{Z})$, the above expression is equal to $-2[L]$. Since $\left(i_{Q}^{*}, i^{*}\right)$ is injective, this implies that (after identifying the cohomology of $y_{\Delta}$ and $\tilde{X}_{0} \cup Q$ ), the class $[\tilde{\Xi}]$ does not lie in the image of $i_{Q, *}$ and hence belongs 
to $\operatorname{coker}\left(i_{Q, *}\right) \hookrightarrow \mathrm{H}^{2 d}(\mathcal{N} ; \mathbb{Z})$. As $\operatorname{deg}(H)$ is odd, the above discussion implies that $2[\tilde{\Xi}] \in \operatorname{Im}\left(i_{Q, *}\right)$ and hence $[\tilde{\Xi}]$ is 2-torsion in $\mathrm{H}^{2 d}(\mathcal{N} ; \mathbb{Z})$.

(ii) Suppose, on the other hand, that $C_{0}$ does not pass through the ordinary double point. Since $\mathcal{C}$ does not pass through the double point of the singular fibre, it is isomorphic to its pullback in $y$. Therefore the support of $\tilde{\Xi}$ has empty intersection with the exceptional fibre $Q$. Thus $\tilde{\Xi}$ goes to zero under the vertical map $\left(i_{Q}^{*} \cdot i^{*}\right)$. This implies that its class in the cohomology of $y$ is zero. Hence its restriction to $\mathcal{N}$ is zero.

\subsection{More Cycles}

We wish now to generalise the above situation to one where there are finitely many cycles on a variety $X$, the generic fibre of a flat family $X \rightarrow S$ with only ordinary double points occurring as singularities. For this we assume that for $i=1, \ldots, l$, there are diagrams:

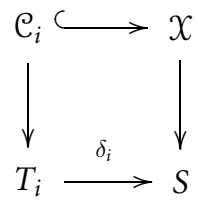

Here $\delta_{i}$ is finite proper map of smooth curves with simple ramification points and such that its branch locus is contained in the singular locus of the family $X \rightarrow S$. Moreover, $\mathrm{C}_{i} \rightarrow T_{i}$ is a smooth family with generic fibre $C_{i}$ which is a codimension $d$ subvariety of $X$.

Let $T:=T_{1} \times_{S} \cdots \times_{S} T_{l}$ and $X_{T} \rightarrow T$ be the base change. Let $y$ denote the blow-up of $X_{T}$ at all its ordinary double points.

Assumption 2.3 The branch loci for the maps $\delta_{i}$ in diagram 2 are distinct. Moreover for each $i, \mathfrak{C}_{i}$ misses the singularities in the fibres over points outside its branch locus and passes through exactly one ordinary double point on each of the singular fibres over the points in the branch locus.

For any integer $1 \leq i \leq l$ one can now construct finitely many diagrams in the following manner: we choose a small open disc $\Delta_{i} \subset S$ containing exactly one branch point of $\delta_{i}$ contained in $S$. Since the various branch loci are disjoint, $\Delta_{i}$ can be so chosen such that it does not contain branch points of $\delta_{j}$ for $j \neq i$. We then have the following diagrams:

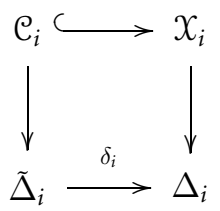


and for $j \neq i$,

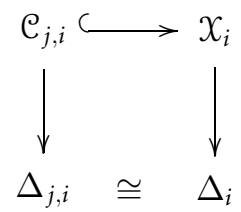

Here the families $\mathcal{C}_{j, i}, \mathcal{C}_{i}, X_{i}$ are the restrictions of the families in diagram (2) to the disc $\Delta_{i}$. As earlier, we may assume without loss of generality that the singular fibres have exactly one ordinary double point.

Thus we have the following situation.

(i) For $j \neq i$ the family $\mathcal{C}_{j, i}$ completely misses the singular locus of the special fibre of the family $x_{i} \rightarrow \Delta_{i}$.

(ii) The map $\tilde{\Delta}_{i} \rightarrow \Delta_{i}$ is a double cover which is ramified at one point.

(iii) For the family of cycles $\mathcal{C}_{i}$, there exists a point $0 \in \Delta_{i}$ such that the special fibre $C_{0}$ at 0 passes through the ordinary double point of the (singular) fibre of $x_{i} \rightarrow \Delta_{i}$.

On a general member $X$ of the family $X_{i} \rightarrow \Delta_{i}$, we then have finitely many codimension $d$ cycles $\xi_{j}=\operatorname{deg}(H) \cdot C_{j}-\operatorname{deg}\left(C_{j}\right) \cdot H$ where $C_{j}$ is a general member of the family $\mathcal{C}_{j, i}$ and $H$ is a codimension $d$ linear section in $X$.

\subsection{Relations Between Cycles}

We now wish to study the relations between the cycles $\tilde{\Xi}_{i}$ defined by $\xi_{i}$ in $X$ as it varies in the family $y \rightarrow T$.

Theorem 2.4 The cycles $\tilde{\Xi}_{i}$ are linearly independent modulo 2 in $\mathrm{CH}^{d}(y)$, the Chow group of codimension d cycles.

Proof Suppose there exists a relation

$$
\sum_{i} n_{i} \tilde{\Xi}_{i}=0 .
$$

If $p: T \rightarrow S$ denotes the morphism between $T$ and $S$, then one notes that for any $i, p^{-1}\left(\Delta_{i}\right) \cong \tilde{\Delta}_{i}$. For $i=i_{0}$, consider the restriction of the above sum to $y_{i_{0}}:=$ $y_{p_{p-1}\left(\Delta_{i}\right)}$. Note that $y_{i_{0}}$ is isomorphic to $X_{i} \times_{\Delta_{i}} \tilde{\Delta}_{i}$ blown up at the ordinary double point occuring in the special fibre of $\mathcal{X}_{i} \rightarrow \Delta_{i}$. Then one has a relation $\sum_{i} n_{i}\left[\tilde{\Xi}_{i}\right]=0$ in $\mathrm{H}^{2 d}\left(y_{i_{0}}, \mathbb{Z}\right)$. Consider its image in the cohomology of $\mathcal{N}_{i_{0}}$ under the map

$$
\mathrm{H}^{2 d}\left(y_{i_{0}}, \mathbb{Z}\right) \rightarrow \mathrm{H}^{2 d}\left(\mathcal{N}_{i_{0}}, \mathbb{Z}\right) .
$$

By Theorem 2.2 we know that $\left[\tilde{\Xi}_{i}\right]$ vanishes for $i \neq i_{0}$ since these do not intersect the exceptional divisors over the singularities in the special fibre of $X_{i_{0}} \rightarrow \Delta_{i_{0}}$. This implies that $n_{i_{0}} \cdot\left[\tilde{\Xi}_{i_{0}}\right]=0$. Since $\left[\tilde{\Xi}_{i_{0}}\right]$ is a non-trivial 2-torsion class, this implies that $n_{i_{0}}$ is divisible by 2 . Similarly arguing, we see that 2 divides $n_{i}$ for all $i$. Hence the cycles are linearly independent modulo 2. 


\subsection{Torsion in Chow Groups}

We shall now make some remarks about the rank of $\mathrm{CH}^{2}(y)$. We make note of the following useful lemma.

Lemma 2.5 If $G$ is an abelian group such that its torsion subgroup $G_{\text {tor }}$ is a subgroup of $((0) / \mathbb{Z})^{r}$, then we have

$$
\operatorname{rank}_{\mathbb{O}}\left(G \otimes(\mathbb{O})+r \geq \operatorname{rank}_{\mathbb{Z} / 2 \mathbb{Z}}(G \otimes \mathbb{Z} / 2 \mathbb{Z})\right.
$$

Proof See [4].

We shall now show that $\mathrm{CH}^{2}(y)_{\text {tors }}$, the subgroup of torsion cycles is a subgroup of $((0) / \mathbb{Z})^{r}$ for some $r$. By the Merkurjev-Suslin theorem [9], one has for any $l$,

$$
\mathrm{CH}^{2}(y)(l) \subset \mathrm{H}_{e t}^{3}\left(y,\left(\mathrm{O}_{l} / \mathbb{Z}_{l}(2)\right)\right.
$$

Taking limits over all $l$, we have

$$
\mathrm{CH}^{2}(y)_{\text {tors }} \subset \mathrm{H}^{3}(y,(\mathrm{O}) / \mathbb{Z}(2)) .
$$

Now,

$$
\begin{aligned}
\mathrm{H}^{3}(y,(\mathbb{O}) / \mathbb{Z}) & \cong \mathrm{H}^{3}(y, \mathbb{Z}) \otimes(\mathbb{O}) / \mathbb{Z} \oplus \mathrm{H}^{4}(y, \mathbb{Z})_{\text {tors }} \\
& \cong((\mathbb{O}) / \mathbb{Z})^{b_{3}} \oplus\left[\bigoplus_{k=1}^{N}\left(\mathbb{Z} / p_{k}^{n_{k}}\right)\right] .
\end{aligned}
$$

The second isomorphism follows from the fact that $\mathrm{H}^{4}(y, \mathbb{Z})$ is of finite type. Since $\mathbb{Z} / p_{k}^{n_{k}} \subset(\mathbb{O}) / \mathbb{Z}$, we finally have the desired inclusion, i.e.,

$$
\mathrm{CH}^{2}(y)_{\text {tors }} \subset((\mathrm{O}) / \mathbb{Z})^{r} \text {. }
$$

Corollary 2.6 Suppose there exist countably infinite number of diagrams as in (1) satisfying the assumptions above. Further assume $d=2$. Then the cycles $\left\{\Xi_{i}\right\}$ generate a subgroup whose rank when tensored with $(\mathrm{O})$ is infinite. In particular, the cycles of Clemens and Paranjape can be detected along with their relations after spreading out.

Proof Let $G \subset \mathrm{CH}^{2}(y)$ be the group generated by the cycles $\tilde{\Xi}_{i}$. Since $G \otimes \mathbb{Z} / 2 \mathbb{Z}$ has infinite rank we conclude from Lemma 2.5 and the discussion preceding this corollary that $G$ has infinite rank.

\section{Conclusions}

We have shown that it is possible to detect almost all known cycles along with their relations in a method similar to the above. The list of such cycles and other details can be found in [11]. 


\section{Acknowledgements}

This note is based on the author's thesis. The author wishes to thank K. Paranjape for suggesting the problem and providing guidance. We also thank M. Nori for pointing out an error and to J. Biswas and G. Dayal for useful discussions. Finally, the author would like to thank the referee for his suggestions which improved the exposition and eliminated an error in section 2.5.

\section{References}

[1] M. Asakura, Motives and algebraic de Rham cohomology. In: The Arithmetic and Geometry of Algebraic Cycles. CRM Proc. Lecture Notes 24, American Mathematical Society, Providence, RI, 2000, pp. 133-154,

[2] J. Biswas, G. Dayal, K. Paranjape and G. V.Ravindra, Higher Abel-Jacobi maps. In: Algebra, Arithmetic and Geometry, Tata Inst. Fund. Res. Stud. Math. 16, Tata Inst. Fund. Res., Bombay, 2002, pp. 159-169.

[3] J.-L. Colliot-Théléne, J.-J.Sansuc and C. Soulé, Torsion dans le groupe de Chow de codimension deux. Duke Math J. 50(1983), no. 3, 763-801.

[4] H. Clemens, Homological equivalence modulo algebraic equivalence is not finitely generated. Inst. Hautes Études Sci. Publ. Math. 58, (1983), 19-38.

[5] H. Esnault and E. Viehweg, Deligne-Beilinson cohomology. In: Beilinson's Conjectures on Special Values of $L$-Functions, Perspect. Math. 4, Academic Press, Boston, MA, 1988, pp. 43-91.

[6] U. Jannsen, Mixed motives, motivic cohomology, and Ext-groups. Proceedings of the International Congress of Mathematicians, Birkhäuser, Basel, 1995, pp. 667-679.

[7] Lecomte, Florence, Rigidité des groupes de Chow. Duke Math. J. 53(1986), no. 2, 405-426.

[8] J. Lewis, A filtration on the Chow groups of a complex projective variety. Compositio Math. 128(2001), no. 3, 299-322.

[9] A. S. Merkurjev, and A. A. Suslin, $K$-cohomology of Severi-Brauer varieties and the norm residue homomorphism. (Russian) Izv. Akad. Nauk SSSR Ser. Mat. 46(1982), no. 5, 1011-1046, $1135-1136$.

[10] K. H. Paranjape, Curves on 3-folds with trivial canonical bundle. Proc. Indian Acad. Sci. Math. Sci. 101(1991) no. 3, 199-213.

[11] G. V. Ravindra, Cohomological Detection of Algebraic Cycles, Thesis submitted to Madras University, 2001.

[12] M. Saito, Filtrations on Chow groups and transcendence degree. Publ. Res. Inst. Math. Sci. 40(2004), no. 3, 933-948.

[13] V. Srinivas, Gysin maps and cycle classes for Hodge cohomology. Proc. Indian Acad. Sci. Math. Sci. 103(1993), no. 3, 209-247.

Department of Mathematics

Washington University

St. Louis, MO 63130

U.S.A.

e-mail: ravindra@math.wustl.edu 